\title{
Diabetes mellitus como factor predictor de tuberculosis en el Servicio de Salud Metropolitano Sur en Santiago, Chile
}

\author{
PAULINA RAMONDA C.*, PAULINA PINO Z.** y LUIS IVÁN VALENZUELA CH.***
}

\section{Diabetes mellitus as a predictor factor of tuberculosis in the Servicio de Salud Metropolitano Sur (SSMS-South Metropolitan Health Service) in Santiago, Chile}

Background: Diabetes mellitus (DM) could be an important factor in identifying people with a high risk of tuberculosis (TB) in Chile, thus, we aim at studying the force of the relationship of these two pathologies. Methods: A case-control study was undertaken using secondary data from the SSMS population group. The cases $(n=473)$ were defined as first episodes of confirmed TB patients 15 years and older, registered between 2006 and 2009. The controls $(n=507)$ were non TB cases, residents in the same Health Service area, randomly selected from the population registered under the public health care system. Risk factors were taken from SIGGES (system for health information and management) files. Logistic regression adjusting for age and social vulnerability was used to estimate the OR $( \pm C I$ 95\%). Results: The higher frequency of DM among cases OR $=3.3$ (95\% CI 2.2-5.0) was confirmed. The inclusion of confounders -age, health insurance and residence in poorer counties- in the model yields produced an adjusted OR $=2.3$ (95\%CI 1.5-3.6). Conclusions: DM is strongly associated with $T B$ in Chilean settings, therefore it may be anticipated that adding this diabetes criteria in screening of $T B$ would help to increase earlier and inclusive diagnosis.

Key words: Tuberculosis, at-risk groups, diabetes mellitus, case-control.

\section{Resumen}

Introducción: La diabetes mellitus (DM) puede ser un importante factor para la identificación de personas con alto riesgo de tuberculosis (TBC) en Chile, por lo que se pretende estudiar la asociación entre estas dos patologías. Métodos: Se efectuó un estudio de caso-control con datos secundarios, obtenidos ambos de la población asignada al Servicio de Salud Metropolitano Sur. Los casos $(n=473)$ son pacientes de 15 o más años con un primer episodio de tuberculosis confirmada, entre los años 2006 y 2009. Los controles $(n=507)$ son residentes del mismo Servicio, pero sin diagnóstico de TBC, seleccionados aleatoriamente de la población de inscritos en el Sistema Público de atención. Los factores de riesgo considerados son los consignados en el sistema de información para la gestión de garantías en salud (SIGGES). La estimación de asociación entre TBC y DM se efectuó mediante regresión logística, ajustando por edad y vulnerabilidad social para la estimación del OR ( \pm IC95\%). Resultados: Se confirma la mayor frecuencia de diabetes mellitus entre los casos, OR = 3,3 (IC95\% 2,2-5,0). La inclusión en el modelo de las variables de confusión: edad, riesgo previsional y residencia en una comuna más pobre que la Región Metropolitana, identifica un estimador (OR) ajustado de 2,3 (IC95\% 1,5-3,6). Conclusiones: La DM se asocia con un mayor riesgo de enfermar de TBC. Orientando intervenciones diagnósticas, con pesquisa dirigida a los diabéticos aumentaría el diagnóstico precoz de la tuberculosis.

Palabras clave: Tuberculosis, grupos de riesgo, diabetes mellitus, caso-control.

\footnotetext{
* Médico neumotisióloga. Servicio de Salud Metropolitano Sur (SSMS).

** Profesora asociada, Escuela de Salud Pública, Facultad de Medicina, Universidad de Chile.

***Matrón, Monitor de Sistema de Información para la Gestión de Garantías Explícitas en Salud. Departamento de Gestión e Información, SSMS.
} 


\section{Introducción}

Desde el año 2000 Chile exhibe el estatus de país con baja prevalencia de tuberculosis, alcanzando en 2010 una incidencia de 13,3/100.000 habitantes ${ }^{1}$. Aunque es un logro destacable para un país en desarrollo, no se alcanzó la etapa de eliminación avanzada (bajo 10/100.000), meta fijada en los objetivos sanitarios para ese año ${ }^{2}$. El rendimiento de la pesquisa pasiva a través del examen diagnóstico, entre los sintomáticos respiratorios que consultan es bajo (cercano al $1 \%)^{3}$. Dada la nueva meta para el decenio (tasa morbilidad de $5 / 100.000)^{4}$ se hace necesario optimizar las estrategias de localización de casos, puesto que en este escenario de baja prevalencia, el diagnóstico de TBC raramente es postulado frente a un paciente que consulta con síntomas respiratorios. La disminución de la incidencia favorece la concentración de la enfermedad en grupos vulnerables por la presencia de factores de riesgo biológicos o sociodemográficos ${ }^{5-14}$. Un ejemplo destacado es el virus de la inmunodeficiencia humana (VIH), determinante de un notable aumento de la probabilidad de enfermar por tuberculosis ${ }^{5,15}$, de tal forma que este conocimiento está totalmente incorporado en la pesquisa de casos. Lo mismo no ocurre con otras enfermedades como la diabetes mellitus, que según se ha descrito incrementa el riesgo de TBC en 2 a 4 veces $^{6,16}$. La edad, que también constituye un factor riesgo, tampoco es considerada como criterio de búsqueda de casos, en circunstancias que los adultos mayores (AM) presentan tasas de morbilidad TBC hasta tres veces más altas ${ }^{17}$, situación que hay que considerar, puesto que la proporción de AM va en aumento (de $8 \%$ a $9,3 \%$ entre 2002 y 2011$)^{18,19}$. Por ello, cada vez es más importante complementar la localización de casos focalizándola en los grupos de mayor riesgo ${ }^{20}$. Datos de 2006 muestran que entre 20 y 55\% de los enfermos de la Región Metropolitana (RM) presentan factores de riesgo, siendo el grupo "adulto mayor" el que alcanza mayor porcentaje, seguido de la condición VIH o síndrome de inmunodeficiencia adquirida (SIDA) ${ }^{21}$.

Otro grupo que ya alcanza importancia poblacional es el de los diabéticos. De acuerdo con la American Diabetes Association (ADA), la DM que afectaba al $8 \%$ de los adultos en Estados Unidos entre los años 1988 y 1994, aumentó al 9,3\% entre 1999 y $2002^{22,23}$. En Chile, la Encuesta Nacional de Salud de Chile (ENS), registró una prevalencia de DM de $6,3 \%$ en $2003^{24,25}$ y de 9,4\% (IC95\% 8,1-10,9) en 2009. Más aún, en los adultos mayores alcanzó un 25,8\% (IC95\% $20,6-31,9)^{26}$.
Frente a esta realidad, es necesario establecer si la relación entre la DM y la TBC es suficientemente importante como para justificar la localización de casos en diabéticos con síntomas respiratorios.

Actualmente, un estudio de este tipo, es posible de ser realizado utilizando la información disponible en los Servicios de Salud. El presente trabajo evalúa -a partir de datos del Servicio de Salud Metropolitano Sur (SSMS)- a nivel poblacional en Chile la asociación entre DM y TBC, con la finalidad de orientar futuras intervenciones diagnósticas que logren una pesquisa precoz de la tuberculosis.

\section{Métodos}

Se efectuó un diseño de caso-control con datos secundarios, obtenidos ambos de la población asignada al Servicio de Salud Metropolitano Sur. La población accesible son los inscritos en el SSMS. En 2009 el SSMS tenía una población asignada de 1.061 .314 habitantes ${ }^{19}$ (proyección Censo del 2002), para ese mismo año el Fondo Nacional de Salud (FONASA), reporta 915.556 inscritos $(86,3 \%)$, distribuidos en 11 comunas con un total de 33 Consultorios y Centros de Salud Familiar $^{27}$.

Los casos son todos los pacientes con un primer episodio de tuberculosis activa, pulmonar o extrapulmonar confirmada, de 15 o más años, diagnosticados entre los años 2006 y 2009, residentes en el SSMS. El Programa de Control y Eliminación de la Tuberculosis (PROCET) incluye a toda la población asignada, con o sin situación previsional al día, tanto del sistema público como del privado. Como en el resto del país, casi la totalidad de los tuberculosos son tratados en la Atención Primaria de Salud (APS), se asume que el grupo estudiado sea representativo del total de casos reales. Se consideró caso confirmado a los pacientes con bacteriología $(+)$ (baciloscopia o cultivo de Koch), o con biopsia (+) de cualquier órgano, con presencia de granulomas caseosos. Se excluyó en este estudio a los pacientes con infección concomitante confirmada del virus de la inmunodeficiencia humana y a los fallecidos con diagnóstico de TBC.

Para la conformación del grupo control (no casos) se utilizó el registro del "Sistema de Información para la Gestión de Garantías en Salud" (SIGGES) ${ }^{28}$. Éste permite ubicar a todas las personas a través del sistema numerado de identificación individual: "rol único nacional" (RUN) si son residentes en el país, independientemente 
de su condición de beneficiarios del sistema por presencia de enfermedades con garantías explícitas en salud (GES). Para la identificación del marco muestral, se obtuvo la base de datos con nombre y RUN ("lista rutificada") de los inscritos de 9 de las 11 comunas que conforman el SSMS*. Se identificó a los casos mediante el RUN a fin de sustraerlos del marco muestral, el cual quedó conformado por los residentes inscritos en el SSMS, sin diagnóstico de tuberculosis. Mediante muestreo aleatorio simple se seleccionó a los controles.

Las pérdidas fueron el $1,7 \%$ de los casos, por información no disponible en el SIGGES debido a RUN omitido o incorrecto. Entre la población referente hubo dos pérdidas por RUN incorrecto $(0,4 \%)$ (Figura 1$)$.

Para los casos y controles la información demográfica, socioeconómica y de otros diagnósticos GES se obtuvo del SIGGES.

Considerando los criterios de inclusión y exclusión finalmente se contó con 473 casos y 507 controles (Figura 1). Con este tamaño de muestra y asumiendo una prevalencia de DM de $16 \%$ en los casos y de 9,4\% en los controles (ENS 2009), el estudio es capaz de detectar un OR de al menos 1,8 con un poder de $95 \%$-con un nivel de confianza también- de $95 \%$.

La variable de efecto es la tuberculosis confirmada. La variable de exposición, es la existencia de un diagnóstico de diabetes mellitus ${ }^{29}$. Las variables confundentes consideradas en este estudio son la edad y el nivel socio-económico (NSE), relacionadas tanto con la DM como con la TBC. Como variable proxy del NSE se utilizó una combinación del sistema previsional y la comuna de residencia, resultando tres categorías: 1 = FONASA "C" o "D" o Institución de Salud Previsional (ISAPRE) y residencia en comuna con menor porcentaje de pobreza que el promedio de la RM (11,5\%). 2 = FONASA “A" o "B" (no recibe ingresos o recibe menos de un salario mínimo) o residencia en comuna con mayor porcentaje de pobreza que el promedio de la RM y $3=$ FONASA "A" o "B" y residencia en comuna con mayor porcentaje de pobreza que el promedio de la RM.

El análisis se orientó a la estimación del $\mathrm{OR} \pm$ $\mathrm{IC}_{95 \%}$ de TBC asociada a la presencia de DM. Mediante un modelo de regresión logística no condicional se obtuvo los valores ajustados por las variables confundentes (edad, situación previsional y procedencia de comuna más vulnerables en relación a la RM). Se utilizó el Programa
STATA $\AA$, versión $11.0^{30}$.

El protocolo fue revisado y aprobado por el Comité de Ética de Investigación Clínica del SSMS en junio 2011. El estudio no significó daños a las personas. Los identificadores individuales fueron omitidos de los archivos, de forma de resguardar la confidencialidad.

\section{Resultados}

\section{Características de los casos de tuberculosis}

La mayor parte de los casos $(86,3 \%)$ fueron confirmados bacteriológicamente (baciloscopia o cultivo de Koch), siendo la localización pulmonar $(83,7 \%)$ la más prevalente; $60,7 \%$ eran de sexo masculino, con una edad mediana de 52 años Recorrido intercuartil (RIC) 36-65 años. El porcentaje de adultos mayores ( $\geq 65$ años) fue de 26,2\% (Tabla 1 ).

Los pacientes TBC con DM presentaron edades más avanzadas (mediana $=58$ años; RIC 51-67) que el grupo TBC sin DM (mediana de edad de 48 años; RIC 32-64) (Mann Whitney: $p<0,001)$. Además, en este grupo con DM la confirmación de TBC con bacteriología $\left(\mathrm{OR}=5,3 ; \mathrm{IC}_{95 \%} 1,6-17,2\right)$ y con baciloscopia $\left(\mathrm{OR}=2,3 ; \mathrm{IC}_{95 \%} 1,3-3,9\right)$ es significativamente más elevada que en el grupo sin DM (Tabla 2).

\section{Características de los casos y los controles}

Los casos fueron de mayor edad (mediana $=52$ años; RIC 36-65) que los controles (mediana de edad de 41 años; RIC 26-54) (Mann Whitney: $\mathrm{p}<0,001)$. El porcentaje de adultos mayores en ambos grupos fue 26,2 y $13 \%$, siendo la diferencia significativa $\left(\mathrm{OR}=2,4\right.$; $\mathrm{IC}_{95} \% 1,7$ $3,3)$, especialmente entre las mujeres $(\mathrm{OR}=3,3$; $\mathrm{IC}_{95 \%}$ 2,1-5,2). La proporción de hombres es mayor entre los casos $\left(\mathrm{OR}=2,0 ; \mathrm{IC}_{95 \%}\right.$ 1,5-2,6). En relación a las variables socio-económicas obtenidas del SIGGES (información no disponible en $7,2 \%$ de los casos y en $8,9 \%$ de los controles), los casos fueron catalogados en tramos más bajos en la situación previsional (FONASA “A” y "B”) que los controles $\left(\mathrm{OR}=2,0 ; \mathrm{IC}_{95} \%\right.$ 1,5-2,7). Los casos también viven más frecuentemente en comunas bajo el promedio de una escala socioeconómica de la Región Metropolitana $(\mathrm{OR}=1,5$; $\mathrm{IC}_{95 \%}$ 1,05-2,1) (Tabla 3).

En la Tabla 4 se presenta la situación de casos y controles en cuanto a la existencia de patologías reconocidamente relacionadas con la tuberculo-

\footnotetext{
*Las comunas que proporcionaron esta información fueron: Buin, Calera de Tango, La Cisterna, Lo Espejo, Paine, Pedro Aguirre Cerda, San Bernardo, San Joaquín y San Miguel.
} 


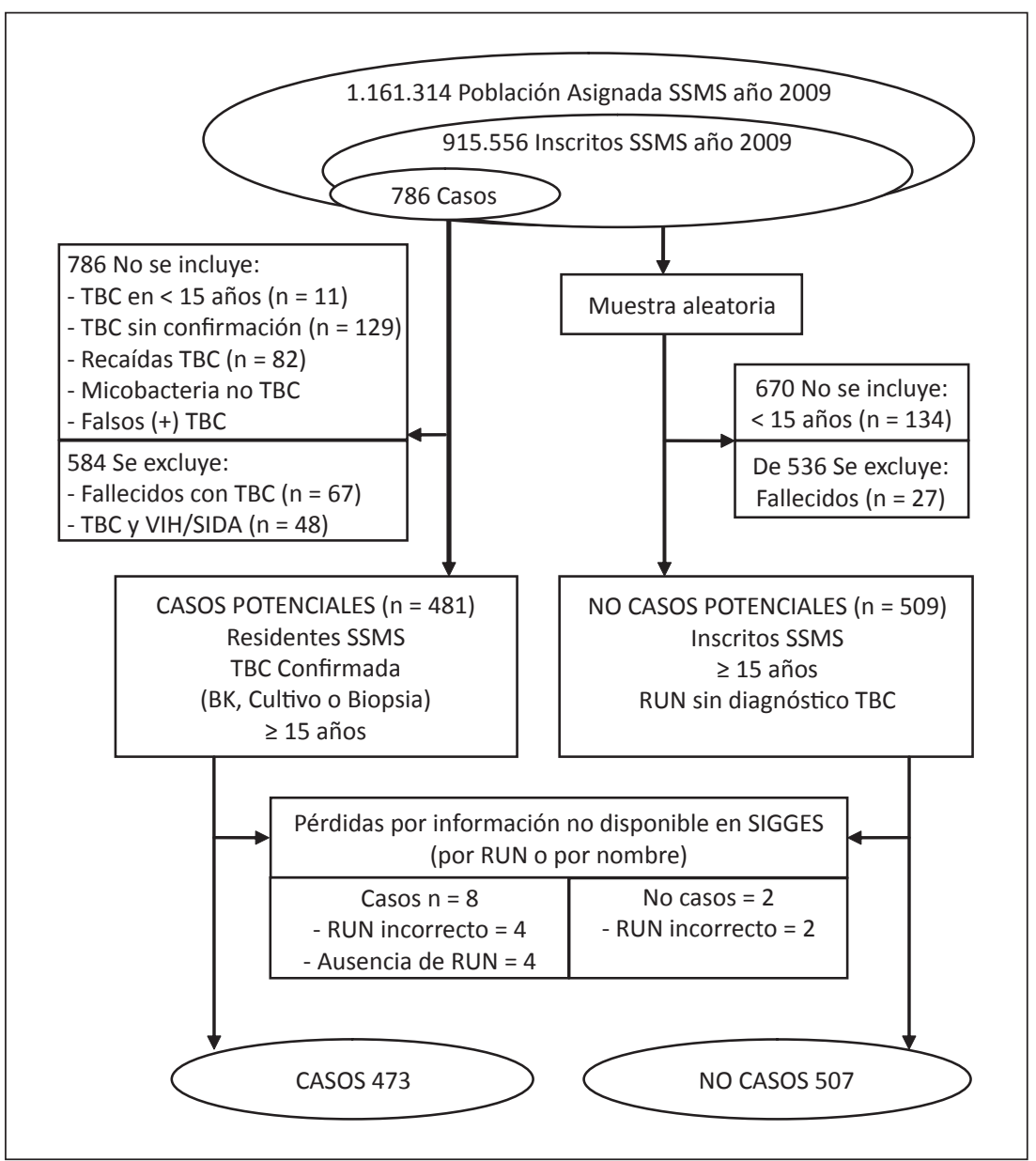

Figura 1. Flujo de selección de casos y controles en el Servicio de Salud Metropolitano Sur, entre los años 2006 y 2009. VIH/SIDA $=$ virus inmuno-deficiencia humana/ síndrome inmuno-deficiencia adquirida; $\mathrm{BK}=$ baciloscopia; SIGGES = sistema de información para la gestión de garantías en salud; RUN = rol único nacional.

Tabla 1. Características de los casos de tuberculosis en el Servicio de Salud Metropolitano Sur entre los años 2006 y 2009

\begin{tabular}{|lrr|}
\hline & $\begin{array}{r}\text { Casos TBC } \\
\text { n (\% o RIC) }\end{array}$ \\
Sexo & & \\
Masculino & 287 & $(60,7)$ \\
Femenino & 186 & $(39,3)$ \\
Edad en años & & \\
Mediana y RIC & 52 & $(36-65)$ \\
Adulto mayor (AM $\geq 65$ años) & 124 & $(26,2)$ \\
Localización TBC & \multicolumn{2}{c}{} \\
Pulmonar & 396 & $(83,7)$ \\
Extrapulmonar & 77 & $(16,3)$ \\
Más de 1 localización & 13 & $(2,8)$ \\
Miliar, meníngea o diseminada & 5 & $(1,1)$ \\
Confirmación TBC & & \\
Baciloscopia & 296 & $(62,6)$ \\
Cultivo de Koch & 112 & $(23,7)$ \\
Bacteriología en conjunto & 408 & $(86,3)$ \\
Biopsia & 65 & $(13,7)$ \\
\hline
\end{tabular}

$\mathrm{TBC}=$ tuberculosis; $\mathrm{RIC}=$ recorrido intercuartílico; $\mathrm{AM}=$ adulto mayor. sis. La disparidad de presentar una patología GES es significativamente mayor en los casos $(\mathrm{OR}=$ 2,3; $\mathrm{IC}_{95 \%}$ 1,8-3,0). Específicamente se confirma la mayor frecuencia de diabetes mellitus entre los casos $\left(\mathrm{OR}=3,3 ; \mathrm{IC}_{95 \%} 2,2-5,0\right)$. Esta relación por cierto, está afectada por la edad, factor que se considerará más adelante.

La enfermedad pulmonar obstructiva crónica (EPOC) y la neumonía del AM estuvieron asociadas con los casos de tuberculosis de manera significativa (EPOC: $\mathrm{OR}=5,1 ; \mathrm{IC}_{95 \%} 2,6-9,9$ y neumonía en $\mathrm{AM}$ : $\left.\mathrm{OR}=5,2 ; \mathrm{IC}_{95 \%} 1,9-14,0\right)$.

Se evaluó el papel del sexo, que aunque está fuertemente relacionado con la TBC, no fue considerado como confusor por no estar relacionado con la diabetes, confirmándose que su inclusión en el modelo logístico no altera la fuerza de la asociación. Según se observa en la Tabla 5, sólo la edad actuó como factor confundente, resultando la asociación entre DM y TBC un OR de 2,3 ( $\mathrm{IC}_{95 \%}$ 1,5-3,6) ajustado por edad y proxy de NSE (riesgo previsional y residencia en una comuna más pobre que la $\mathrm{RM}$ ). 
Tabla 2. Características de pacientes tuberculosos según presencia de diabetes mellitus en el Servicio de Salud Metropolitano Sur entre los años 2006 y 2009

\begin{tabular}{|c|c|c|c|}
\hline Características & $\begin{array}{c}\text { TBC con DM } \\
\text { n }(\%)\end{array}$ & $\begin{array}{c}\text { TBC } \sin \mathrm{DM} \\
\text { n }(\%)\end{array}$ & OR (IC 95\%) \\
\hline Edad en años Mediana y RIC & 58 (RIC 51-67) & 48 (RIC 32-64)* & \\
\hline $\begin{array}{l}\text { Sexo } \\
\text { Razón H:M } \\
\text { Razón Masculinidad }\end{array}$ & $\begin{array}{r}48 / 38 \\
126,3\end{array}$ & $\begin{array}{c}239 / 148 \\
161,5\end{array}$ & $0,8(0,5-1,3)$ \\
\hline $\begin{array}{l}\text { Localización } \\
\text { TBC Pulmonar } \\
\text { TBC solo EP } \\
\text { TBC Pulmonar y EP } \\
\text { TBC más de una localización } \\
\text { TBC Miliar, meníngea o diseminada }\end{array}$ & $\begin{array}{rr}78 & (90,7) \\
8 & (9,3) \\
2 & (2,3) \\
2 & (2,3) \\
0 & (0,0)\end{array}$ & 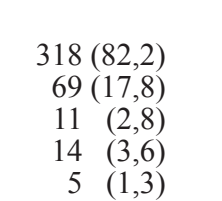 & $\begin{array}{c}2,1(0,98-4,6) \\
4,7(0,2-1,0) \\
0,8(0,2-3,8) \\
0,6(0,1-2,9) \\
\text { No calculable }\end{array}$ \\
\hline $\begin{array}{l}\text { Confirmación de la TBC } \\
\text { Baciloscopia } \\
\text { Cultivo de Koch } \\
\text { Bacteriología en conjunto } \\
\text { Biopsia }\end{array}$ & $\begin{array}{r}66(76,7) \\
17(19,8) \\
83(96,5) \\
3(3,5)\end{array}$ & $\begin{array}{r}230(59,4) \\
95(24,6) \\
325(84,0) \\
62(16,0)\end{array}$ & $\begin{array}{l}2,3(1,3-3,9) \\
0,8(0,4-1,4) \\
5,3(1,6-17,2) \\
0,2(0,1-0,6)\end{array}$ \\
\hline $\begin{array}{l}\text { Factores socio-económicos } \\
\text { FONASA A o B } \\
\text { PRAIS } \\
\text { Comuna más pobre que RM } \\
\text { Comuna más pobre que país }\end{array}$ & $\begin{array}{r}64(78,1) \\
4(4,7) \\
81(94,2) \\
36(41,9)\end{array}$ & $\begin{array}{r}274(76,8) \\
15(3,9) \\
329(85,0) \\
146(37,3)\end{array}$ & $\begin{array}{l}1,1(0,6-1,9) \\
1,2(0,4-3,7) \\
2,9(1,1-7,4) \\
1,2(0,7-1,9)\end{array}$ \\
\hline $\begin{array}{l}\text { Co-morbilidad GES respiratoria } \\
\text { EPOC } \\
\text { Asma } \\
\text { NAC en AM }\end{array}$ & $\begin{array}{r}10(11,6) \\
0 \quad(0,0) \\
7(24,1)\end{array}$ & $\begin{array}{rr}38 & (9,8) \\
9 & (2,3) \\
30 & (31,6)\end{array}$ & $\begin{array}{c}1,2(0,6-2,5) \\
\text { No calculable } \\
0,7(0,3-1,8)\end{array}$ \\
\hline $\begin{array}{l}\text { Otras Co-morbilidades GES } \\
\text { IRC terminal } \\
\text { Cáncer } \\
\text { Depresión }\end{array}$ & $\begin{array}{rr}2 & (2,3) \\
4 & (4,7) \\
10 & (11,6)\end{array}$ & $\begin{array}{rr}1 & (0,3) \\
7 & (1,8) \\
28 & (7,2)\end{array}$ & $\begin{array}{l}9,2(0,8-102,5) \\
2,6(0,8-9,3) \\
1,7(0,8-3,6)\end{array}$ \\
\hline
\end{tabular}

*Mann Whitney $\mathrm{p}<0,001 . \mathrm{TBC}=$ tuberculosis; $\mathrm{RIC}=$ recorrido intercuartílico; $\mathrm{OR}=$ odds ratio; IC $=$ intervalo de confianza; $\mathrm{H}=$ hombre; $\mathrm{M}$ = mujer; $\mathrm{EP}=$ extrapulmonar; FONASA = Fondo nacional de salud; PRAIS = Programa de reparación y atención integral en salud y derechos humanos; RM = Región metropolitana; GES = garantía explícita en salud; EPOC = enfermedad pulmonar obstructiva crónica; NAC en AM = neumonía del adulto mayor adquirida en la comunidad; IRC terminal = insuficiencia renal crónica terminal.

Tabla 3. Características generales de casos y controles en el Servicio de Salud Metropolitano Sur

\begin{tabular}{|c|c|c|c|}
\hline & $\begin{array}{c}\text { Casos TBC } \\
\text { n (\% o RIC) }\end{array}$ & $\begin{array}{c}\text { No casos } \\
\text { n }(\% \text { o RIC })\end{array}$ & $\begin{array}{c}\text { OR Crudo } \\
\text { (95\% IC) }\end{array}$ \\
\hline $\begin{array}{l}\text { Edad en años } \\
\text { Mediana y RIC } \\
\text { Adulto mayor (AM } \geq 65 \text { años) } \\
\text { Porcentaje de AM en hombres } \\
\text { Porcentaje de AM en mujeres }\end{array}$ & $\begin{array}{r}52(36-65) \\
124(26,2) \\
60(21,0) \\
64(34,4)\end{array}$ & $\begin{array}{l}41(26-54)^{*} \\
66(13,0) \\
27(12,2) \\
39(13,7)\end{array}$ & $\begin{array}{l}2,4(1,7-3,3) \\
1,9(1,2-3,1) \\
3,3(2,1-5,2)\end{array}$ \\
\hline $\begin{array}{l}\text { Sexo } \\
\text { Masculino } \\
\text { Femenino } \\
\text { Razón H:M } \\
\text { Razón Masculinidad }\end{array}$ & $\begin{array}{c}287(60,7) \\
186(39,3) \\
287 / 186 \\
154,3\end{array}$ & $\begin{array}{c}222(43,8) \\
285(56,2) \\
222 / 285 \\
77,9\end{array}$ & $2,0(1,5-2,6)$ \\
\hline $\begin{array}{l}\text { Factores socio-demográficos } \\
\text { FONASA A o B } \\
\text { PRAIS } \\
\text { De comuna más pobre } \\
\text { en relación al país } \\
\text { en relación a la RM }\end{array}$ & $\begin{array}{r}338(77,0) \\
19(4,0)\end{array}$ & $\begin{array}{r}288(62,3) \\
22 \quad(4,3)\end{array}$ & $\begin{array}{l}2,0(1,5-2,7) \\
0,9(0,5-1,7)\end{array}$ \\
\hline
\end{tabular}

*Mann Withney $\mathrm{p}<0,001 . \mathrm{TBC}=$ tuberculosis; $\mathrm{RIC}=$ recorrido intercuartílico; $\mathrm{OR}=$ odds ratio; IC $=$ intervalo de confianza; $\mathrm{AM}=$ adulto mayor; $\mathrm{H}=$ hombre; $\mathrm{M}=$ mujer; FONASA = Fondo nacional de salud; PRAIS = Programa de reparación y atención integral en salud y derechos humanos; $\mathrm{RM}=$ Región metropolitana. 
Tabla 4. Diabetes mellitus y patologías GES como factores de riesgo de tuberculosis en el Servicio de Salud Metropolitano Sur

\begin{tabular}{|c|c|c|c|}
\hline & $\begin{array}{c}\text { Casos TBC } \\
\text { n }(\%)\end{array}$ & $\begin{array}{c}\text { No casos } \\
\text { n }(\%)\end{array}$ & $\begin{array}{l}\text { OR Crudo } \\
\text { (95\% IC) }\end{array}$ \\
\hline Con patología GES & $241(51,0)$ & $157(31,0)$ & $2,3(1,8-3,0)$ \\
\hline Diabetes mellitus (DM) & $86(18,2)$ & $32 \quad(6,3)$ & $3,3(2,2-5,0)$ \\
\hline $\begin{array}{l}\text { Patología respiratoria GES } \\
\text { EPOC } \\
\text { Asma } \\
\text { NAC en AM }\end{array}$ & $\begin{array}{r}48(10,2) \\
9(1,9) \\
37(29,8)\end{array}$ & $\begin{array}{rr}11 & (2,2) \\
4 & (0,8) \\
5 & (7,6)\end{array}$ & $\begin{array}{l}5,1(2,6-9.9) \\
2,4(0,7-8,0) \\
5,2(1,9-14,0)\end{array}$ \\
\hline $\begin{array}{l}\text { Otras patologías GES } \\
\text { IRC terminal } \\
\text { Analgesia del parto } \\
\text { Alivio del dolor } \\
\text { Cáncer } \\
\text { Depresión }\end{array}$ & $\begin{aligned} 3 & (0,6) \\
15 & (3,2) \\
2 & (0,4) \\
11 & (2,3) \\
38 & (8,0)\end{aligned}$ & $\begin{aligned} 0 & (0,0) \\
15 & (3,0) \\
0 & (0,0) \\
5 & (1,0) \\
46 & (9,1)\end{aligned}$ & $\begin{array}{l}\text { No calculable } \\
1,1(0,5-2,2) \\
\text { No calculable } \\
2,4(0,8-6,9) \\
0,9(0,6-1,4)\end{array}$ \\
\hline
\end{tabular}

$\mathrm{TBC}=$ tuberculosis; OR = odds ratio; $\mathrm{IC}=$ intervalo de confianza; GES = garantía explícita en salud; EPOC = enfermedad pulmonar obstructiva crónica; NAC en AM = neumonía del adulto mayor adquirida en la comunidad; IRC terminal $=$ insuficiencia renal crónica terminal.

Tabla 5. Asociación* no ajustada y ajustada entre diabetes mellitus y tuberculosis en el Servicio de Salud Metropolitano Sur

\begin{tabular}{|lcc|}
\hline Variable & $\begin{array}{c}\text { OR No ajustado } \\
\mathbf{( 9 5 \% I C )}\end{array}$ & $\begin{array}{c}\text { OR Ajustado } \\
\mathbf{( 9 5 \% I C )}\end{array}$ \\
\hline Diabetes mellitus & $3,3(2,2-5,1)$ & $2,3(1,5-3,6)$ \\
Edad (años) & $1,02(1,02-1,03)$ & $1,02(1,01-1,03)$ \\
Proxy NSE & & \\
Categoría 1 & Referencia & Referencia \\
Categoría 2 & $1,5(0,8-2,7)$ & $1,4(0,8-2,7)$ \\
Categoría 3 & $2,7(1,5-4,9)$ & $2,4(1,3-4,3)$ \\
\hline
\end{tabular}

*Modelo de regresión logística. $\mathrm{OR}=$ odds ratio; $\mathrm{IC}=$ intervalo de confianza; NSE = nivel socio-económico. $\mathrm{n}$ $=473$ casos y 507 no casos, no pareados.

\section{Discusión}

En este estudio de caso-control se ha encontrado una fuerte asociación $\left(\mathrm{OR}=2,3 ; \mathrm{IC}_{95 \%} 1,5\right.$ $3,6)$ entre TBC y DM en el SSMS, donde reside el $6,5 \%$ de la población del país, confirmando hallazgos similares descritos en la literatura internacional. En Chile hemos examinado esta asociación en una población usuaria de un Servicio de Salud de la Región Metropolitana, en un momento que el país presenta baja prevalencia de tuberculosis y prevalencia creciente de diabetes mellitus ${ }^{1,24,26}$. En un estudio de cohorte histórica en 1.529 diabéticos seguidos entre 1959 y 1982 en la VIII Región, (cuando la tasa de incidencia de TBC regional estaba sobre los 77/100.000), Olmos y cols. encontraron que la probabilidad de tener un episodio de TBC durante los primeros 10 años de seguimiento era de 5,9\% para el total de DM: 24\% en DM insulino-dependientes (IDDM) y 4,8\% en DM no insulino-dependientes (NIDDM), con un riesgo de tuberculosis 3,9 veces mayor (valor no ajustado) en los NIDDM que el de la población de 40 años y más ${ }^{16}$.

Se ha establecido que la diabetes es un factor de riesgo para desarrollar infecciones como la tuberculosis, lo que se atribuye a una declinación de la respuesta inmune, es decir, la existencia de una vía común en la historia natural de ambas enfermedades. La diabetes afecta especialmente a células fagocíticas: los macrófagos (y sus precursores los monocitos) y a los linfocitos ${ }^{31}$. Se postula que inicialmente tanto la hiperglicemia como los menores niveles de insulina contribuyen a disminuir directamente el control sobre la carga bacilar. Se afecta la quimiotaxis, la fagocitosis, la activación y la presentación de los antígenos ${ }^{31}$. Hay también una menor actividad bactericida de los leucocitos (macrófagos alveolares menos activos), con menor potencial oxidativo destructivo de los neutrófilos y una menor producción de interferón- $\gamma(\mathrm{IFN}-\gamma)$ lo que traduce una menor respuesta inmune de adaptación de linfocitos $\mathrm{T}$ helper 1 (Th1) ${ }^{32}$ (Figura 2). Varios estudios y en las revisiones publicadas por Dooley y Chaisson en 2009 y Jeon y Murray en 2008, evidencian estimadores de riesgos $\geq 2,0$; especialmente en los países con menores ingresos, entre los diabéticos más severos o con peor control de su enfermedad $y$ en $\operatorname{IDDM}^{7,8,13,31,32}$.

Comparando los casos de TBC con y sin DM destaca la mayor edad en el primer grupo, con TBC pulmonar confirmada en mayor proporción con la bacteriología (lo que implica mayor 


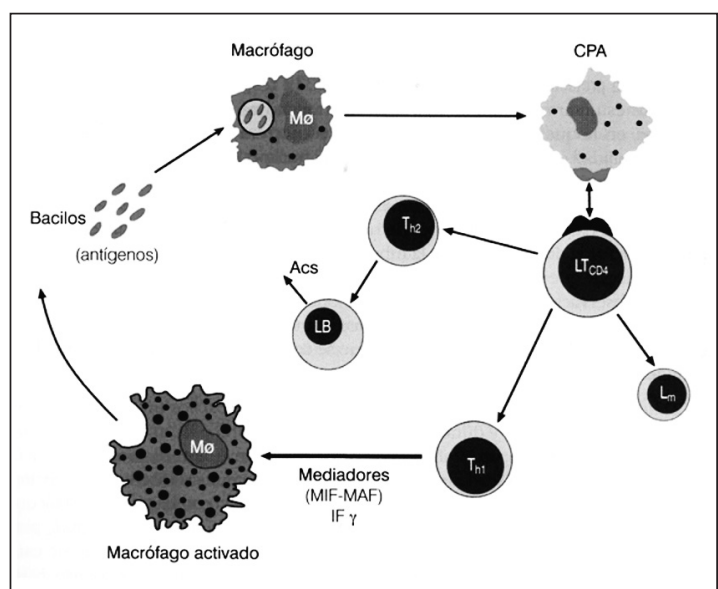

Figura 2. Esquema de la respuesta inmune celular en tuberculosis. CPA: células presentadoras de antígeno; LT CD4: linfocito T helper; Th2: linfocito de la inmunidad humoral; LB: linfocito B; Acs: anticuerpos; Th1: linfocitos de la inmunidad celular; Lm: linfocitos de memoria; MIF: factor inhibidor de la migración de macrófagos; MAF: factor activador del macrófago; IF $\gamma$ : interferón gamma. Reproducción autorizada por los autores ${ }^{15}$.

contagiosidad). Se ha planteado en numerosos estudios que la DM podría determinar una entidad clínica tuberculosa diferente ${ }^{33-37}$. Si bien se pueden agregar algunas particularidades en las imágenes radiológicas, con lesiones más diseminadas y nódulos cavitarios que también incluyen las bases pulmonares ${ }^{37}$, alternativamente se puede postular que habría un diagnóstico tardío de tuberculosis, debido a la inusual sospecha de esta enfermedad entre los diabéticos y también en los adultos mayores, que frecuentemente tienen otras morbilidades.

Una debilidad del estudio es que no pudo obtener el tiempo de evolución de los síntomas antes del diagnóstico de $\mathrm{TBC}$, ni la duración promedio de la diabetes, por lo que no fue posible establecer si la DM precedía a la tuberculosis, un problema inherente a los estudios retrospectivos. Por lo mismo, no se puede descartar la ocurrencia de un sesgo de causalidad reversa, descrita para una minoría de los pacientes TBC (menos del $4 \%$ ), que podrían desarrollar hiperglicemia, a través de un proceso inflamatorio que favoreciera la resistencia insulínica, pero que finalmente logran normalizar su glicemia al comenzar el tratamiento contra la tuberculosis ${ }^{13}$.

En este estudio se buscó validez interna, con grupos comparables y representativos de la población del SSMS. Según la Encuesta de Caracterización Socio-Económica Nacional (CASEN) aplicada el año 2009, tres de las once comunas (La Granja, San Bernardo y Lo Espejo) presen- taban más pobreza que el promedio nacional y otras cinco (San Joaquín, La Cisterna, El Bosque, Pedro Aguirre Cerda y Paine) quedaron clasificadas bajo el porcentaje regional ${ }^{38}$.

Aunque no se tuvo la posibilidad de encuestar o tomar exámenes de sangre que confirmaran el diagnóstico de diabetes mellitus, la búsqueda de los factores de exposición se realizó de la misma manera entre los casos y los controles, por lo que no se espera un sesgo de información diferencial entre ambos grupos.

Las patologías del ámbito respiratorio como la EPOC y la neumonía del AM, estuvieron asociadas con los casos de tuberculosis de manera significativa, lo que permitiría postular que estos diagnósticos enmascaran casos de TBC, dado que los síntomas y signos no son específicos. En un estudio de tuberculosis del Servicio de Salud Metropolitano Sur Oriente en 2004, se evidenció que al momento del diagnóstico el 50\% de los casos TBC pulmonares demostrados por bacteriología ya se encontraba en control médico por otras patologías; 29\% estaba en el Programa Cardiovascular (que incluye el control de la DM) y $21 \%$ en el Programa de Enfermedades Respiratorias del Adulto (ERA) ${ }^{39}$. Por otra parte Leung y cols. ${ }^{40}$, en un estudio de cohorte realizado en Hong Kong, mostraron que cuando la diabetes mellitus estaba bien controlada no aumentó el riesgo de tuberculosis, observación que refuerza el papel causal de la DM y que releva la necesidad de trabajar permanentemente de manera coordinada en el diagnóstico y control de ambas patologías. Una fortaleza del diseño utilizado en este estudio, fue la configuración de los casos sólo con personas cursando su primer episodio de $\mathrm{TBC}$, lo que permite estimar mejor la asociación de DM y TBC, ya que el factor diabetes mellitus no aparece duplicado en las recaídas de tuberculosis.

El estudio se basó en fuentes secundarias, lo que aumenta el riesgo de sesgo, especialmente de información. El ingreso al estudio de los controles no fue pareado por edad, ni hubo restricciones generales previas por las otras posibles variables que conceptualmente pudieran confundir la relación entre la DM y la tuberculosis (edad, NSE). Para el ajuste de confusión se recurrió a un modelo de regresión logística, verificándose que sólo la edad actuaba como factor confundente -disminuyendo el estimador crudo tras su inclusión en el modelo.

No se puede descartar la existencia de confusión residual por no contar con todos los posibles factores confundentes, entre los que se destaca el status nutricional, ya que aunque actualmente en el país es bajo el enflaquecimiento, éste es más 
frecuente en los adultos mayores ${ }^{41}$. Otra potencial fuente de confusión residual deriva de la posible insuficiencia del nivel socio-económico, que sólo se estimó en base al nivel de pobreza de la comuna de residencia y a la situación previsional individual; siendo que ésta no estuvo disponible en algo menos del 10\% de los sujetos y porque la importancia de esta variable es incontestable; Tekkel y cols., en un estudio de caso-control en Estonia en 2002, encontraron que el principal factor de riesgo de tuberculosis no fue la inmigración ni el uso de drogas, sino que el bajo nivel socioeconómico ${ }^{9}$. A pesar de estas limitantes, la pérdida de información para las demás variables fue mínima. El estimador obtenido es por lo demás, bastante semejante a lo reportado en la literatura internacional, mostrando que incluso un estudio basado en registros administrativos existentes puede ser de gran utilidad para la investigación en el país. Por esto, se destaca la importancia de contar con adecuadas bases de datos, con la información requerida recolectada de manera sistemática y confiable, a fin de maximizar el provecho que por su representatividad pueden alcanzar estas bases de datos. Al ser utilizadas tanto para la vigilancia como para la investigación, es posible monitorear sus limitaciones y fortalezas. Así, como ya ocurre en los países más desarrollados, se conocerían de mejor manera los procesos que afectan a la población nacional.

\section{Conclusiones}

La diabetes mellitus se asocia con un mayor riesgo de enfermar de tuberculosis, lo que tiene implicancias clínicas y epidemiológicas. Puesto que en Chile la DM es una patología GES y cerca del $80 \%$ conoce su condición de enfermo ${ }^{26}$, se hace recomendable implementar el tamizaje con pesquisa dirigida a este grupo creciente de la población, para lograr un diagnóstico precoz de TBC.

Como el PROCET mantiene una capacitación continua de sus equipos, porque se requiere un manejo riguroso y un diagnóstico de situación preciso $^{42}$, se propone que los registros incluyan la información referente a la diabetes mellitus. Igualmente se requiere información más completa relacionada con el nivel socio-económico.

\section{Agradecimientos}

Los autores agradecen a los Directores de Salud Comunal del SSMS por su apoyo en la obtención de las listas de los pacientes inscritos en los establecimientos de atención.

\section{Bibliografía}

1.- ZÚÑIGA M. La eliminación de la tuberculosis como problema de Salud Pública. Situación de Chile en el año 2008. Rev Chil Enf Respir 2009; 25: 117-26.

2.- MINSAL, 2002. Los Objetivos Sanitarios para Chile 2000-2010. (Consultada 28 de junio, 2012), at http:// epi.minsal.cl/epi/html/elvigia/vigia15.pdf

3.- LUELMO F. ¿Qué papel cumple la baciloscopia en el esputo en quienes acuden a los centros de salud? In: Toman K, ed. Tuberculosis Detección de casos, tratamiento y vigilancia. Washington, D.C.: OPS. Frieden, T; 2006: 8-11.

4.- MINSAL, 2011. Objetivos Sanitarios 2011-2020. (Consultada 28 de junio, 2012), at http://www.slideshare.net/ CesfamMTC/objetivos-sanitarios-decada-2011-2020.

5.- RIEDER H. Bases epidemiológicas del control de la tuberculosis. París, Francia: Unión Internacional Contra la Tuberculosis y Enfermedades Respiratorias; 1999.

6.- LADEMARCO M, REICHLER M. ¿Qué papel desempeñan los factores del huésped en la patogenia, la prevención y el tratamiento de la tuberculosis? In: Toman $\mathrm{K}$, ed. Tuberculosis Detección de casos, tratamiento y vigilancia. $2^{\mathrm{a}}$ ed. Washington D.C.: OPS. Frieden, T; 2006: 119-23.

7.- SHETTY N, SHEMKO M, VAZ M, D'SOUZA G. An epidemiological evaluation of risk factors for tuberculosis in South India: a matched case control study. Int J Tuberc Lung Dis 2006; 10: 80-6.

8.- COKER R, MCKEE M, ATUN R, DIMITROVA B, DODONOVA E, KUZNETSOV S, et al. Risk factors for pulmonary tuberculosis in Russia: case-control study. BMJ 2006; 332: 85-7.

9.- TEKKEL M, RAHU M, LOIT H M, BABURIN A. Risk factors for pulmonary tuberculosis in Estonia. Int J Tuberc Lung Dis 2002; 6: 887-94.

10.- MINSAL. Perfil epidemiológico básico de la población aymara de la Provincia de Iquique. Santiago, Chile: Ministerio de Salud de Chile; 2007.

11.- YÁÑEZ A. Tuberculosis en inmigrantes. Situación Chile-Perú. Rev Chil Enf Respir 2010; 26: 161-4.

12.- LOBATO M N, MOHAMED M H, HADLER J L. Tuberculosis in a low-incidence US area: local consequences of global disruptions. Int $\mathbf{J}$ Tuberc Lung Dis 2008; 12: 506-12.

13.- ALISJAHBANA B, VAN CREVEL R, SAHIRATMADJA E, DEN HEIJER M, MAYA A, ISTRIANA E, et al. Diabetes mellitus is strongly associated with tuberculosis in Indonesia. Int J Tuberc Lung Dis 2006; 10 : 696-700.

14.- FANG H C, LEE P T, CHEN C L, WU M J, CHOU K J, CHUNG H M. Tuberculosis in patients with end-stage 
renal disease. Int J Tuberc Lung Dis 2004; 8: 92-7.

15.- FARGA V, CAMINERO J A. Tuberculosis. 3ra ed. Santiago, Chile: Editorial Mediterráneo Ltda; 2011.

16.- OLMOS P, DONOSO M, ROJAS N, LANDEROS P, SCHURMANN R, RETAMAL G, et al. Tuberculosis y diabetes mellitus: Estudio longitudinal-retrospectivo en un hospital docente. Rev Med Chile 1989; 117: 979-83.

17.- PEÑA C, GARCÍA C, CHIHUAILAF L. Situación epidemiológica de la Región Metropolitana. Rev Chil Enf Respir 2010; 26: 40-6.

18.- INE, (Chile). Censo 2002: Resultados. Santiago: INE; 2003.

19.- INE, 2011. Proyecciones de población. (Consultado: 20 de junio, 2011), en http://www.ine.cl/canales/chile_estadistico/demografia_y_vitales/demo_y_vita.php.

20.- GARCÍA C. Tuberculosis en los grupos de riesgo de la Región Metropolitana, 2008. Rev Chil Enf Respir 2010; 26: $105-11$.

21.- PEÑA C, TORRES Z. Resumen de actividades del Comité de tuberculosis Región Metropolitana 2007. Rev Chil Enf Respir 2008; 24: 153-8.

22.- Prevalence of Diabetes and Impaired Fasting Glucose in Adults in the U.S. Population. National Health and Nutrition Examination Survey 1999-2002. Diabetes Care, 2006. (Consultado: 1 de agosto, 2011), en http:// care.diabetesjournals.org/content/29/6/1263.full

23.- CDC. National Diabetes Fact Sheet: national estimates and general information on diabetes and prediabetes in the United States, 2011. Atlanta, GA: U.S.: Department of Health and Human Services, Centers for Disease Control and Prevention; 2011.

24.- MINSAL. Gobierno de Chile. Resultado I Encuesta de Salud, Chile 2003.

25.- OPS. Salud de Las Américas. Washington: Volumen II-Países; 2007.

26.- MINSAL. Gobierno de Chile. Resultados Encuesta de Salud ENS, Chile 2009-2010.

27.- FONASA. (Consultado: 13 de abril, 2011), en http:// www.fonasa.cl/.

28.- SIGGES. Sistema de información para la gestión de garantías en salud. (Consultado: 13 de abril, 2011), en http://www.sigges.cl/jsp/menu/homepage.

29.- MINSAL. Guía Clínica Diabetes Mellitus Tipo 2. Santiago, Chile: MINSAL; 2009.

30.- StataCorp LP. Stata Statistical Software. In. 11 ed. College Station TX; 2009.
31.- DOOLEY K E, CHAISSON R E. Tuberculosis and diabetes mellitus: convergence of two epidemics. Lancet Infect Dis 2009; 9: 737-46.

32.- JEON C Y, MURRAY M B. Diabetes mellitus increases the risk of active tuberculosis: a systematic review of 13 observational studies. PLoS Med 2008; 5: e152.

33.- WANG J Y, LEE L N, HSUEH P R. Factors changing the manifestation of pulmonary tuberculosis. Int J Tuberc Lung Dis 2005; 9: 777-83.

34.- BALDE N M, CAMARAA, CAMARA L M, DIALLO M M, KAKE A, BAH-SOW O Y. Associated tuberculosis and diabetes in Conakry, Guinea: prevalence and clinical characteristics. Int J Tuberc Lung Dis 2006; 10: 1036-40.

35.- ALISJAHBANA B, SAHIRATMADJA E, NELWAN E J, PURWA AM, AHMAD Y, OTTENHOFF T H, et al. The effect of type 2 diabetes mellitus on the presentation and treatment response of pulmonary tuberculosis. Clinical Infectious Diseases 2007; 45: 428-35.

36.- PÉREZ-GUZMÁN C, VARGAS M H, TORRESCRUZ A, PÉREZ-PADILLA J R, FURUYA M E Y, VILLARREAL-VELARDE H. Diabetes modifies the male:female ratio in pulmonary tuberculosis. Int $\mathrm{J}$ Tuberc Lung Dis 2003; 7 : 354-8.

37.- PÉREZ-GUZMÁN C, TORRES-CRUZ A, VILLARREAL-VELARDE H, SALAZAR-LEZAMA M A, VARGAS M H. Atypical radiological images of pulmonary tuberculosis in 192 diabetic patients: a comparative study. Int J Tuberc Lung Dis 2001; 5: 455-61.

38.- MIDEPLAN, Chile. Encuesta de Caracterización Socioeconómica Nacional; 2003.

39.- TABILO F, PEÑA C. Reorientación del Programa de Localización de Casos de Tuberculosis. Región Metropolitana, 2006. Rev Chil Enf Respir 2007; 23: 135-40.

40.- LEUNG C C, LAM T H, CHAN W M, YEW W W, HO K S, LEUNG G M, et al. Diabetic control and risk of tuberculosis: a cohort study. Am J Epidemiol 2008; 167 : 1486-94.

41.- ENCA, 2010. Encuesta Nacional de Consumo Alimentario. Universidad de Chile. Datos inéditos; 2011. Ministerio de Salud. (Accessed 21 de julio, 2011), en http://www.redsalud.gov.cl/noticias/noticias.php?id $\mathrm{n}=954 \&$ show $=10-2010$.

42.- ZÚÑIGA M, MARCONE P, RIQUELME C. Reflexiones sobre la tuberculosis en Chile. Rev Chil Enf Respir 2006; 23: 59-66.

Correspondencia a:

Dra. Paulina Ramonda Celedón.

Departamento Técnico en Salud del SSMS

Santa Rosa 3453. San Miguel. Santiago, Chile.

Teléfono: 25763737, fax: 25763726

E-mail: paulina.ramonda@redsalud.gov.cl 